PROCEEDINGS OF THE

AMERICAN MATHEMATICAL SOCIETY

Volume 135, Number 2, February 2007, Pages 383-391

S 0002-9939(06)08465-6

Article electronically published on August 2, 2006

\title{
ON THE ABSENCE OF RAPIDLY DECAYING SOLUTIONS FOR PARABOLIC OPERATORS WHOSE COEFFICIENTS ARE NON-LIPSCHITZ CONTINUOUS IN TIME
}

\author{
DANIELE DEL SANTO AND MARTINO PRIZZI
}

(Communicated by David S. Tartakoff)

\begin{abstract}
We find minimal regularity conditions on the coefficients of a parabolic operator, ensuring that no nontrivial solution tends to zero faster than any exponential.
\end{abstract}

\section{INTRODUCTION, STATEMENTS AND REMARKS}

Let $A$ be a nonnegative self-adjoint operator in a Hilbert space $H$. Consider the Cauchy problem

$$
\left\{\begin{array}{l}
\frac{d u}{d t}+A u=0 \\
u(0)=u_{0}
\end{array}\right.
$$

The solution $u(t)$ can be represented in terms of the spectral resolution $E_{\lambda}$ of $-A$, and it turns out that its asymptotic behavior is like $e^{-\lambda_{0} t}$, where $\lambda_{0}$ is the infimum of those values of $\lambda$ for which $E_{\lambda} u_{0}=u_{0}$. It follows that no solution, except the trivial one, can tend to zero faster than any exponential.

Peter Lax [4] considered nonautonomous perturbations of (1.1) of the form

$$
\left\{\begin{array}{l}
\frac{d u}{d t}+(A+K(t)) u=0 \\
u(0)=u_{0}
\end{array}\right.
$$

where $K(t)$ is a bounded linear operator. He proved that, if the norm of $K(t)$ is sufficiently small, then again solutions of (1.2), unless identically zero, do not tend to zero faster than any exponential.

The question then arised naturally of whether a similar result could hold even for perturbations which were not relatively bounded with respect to $A$. In the years following, attention focussed mainly on parabolic inequalities, written in integrated form, like

$$
\int\left|\partial_{t} u-\sum_{i j} a_{i j}(t, x) \partial_{x_{i}} \partial_{x_{j}} u\right|^{2} d x \leq C_{1}(t) \int|u|^{2} d x+C_{2}(t) \int \sum_{i}\left|\partial_{x_{i}} u\right|^{2} d x
$$

Received by the editors September 7, 2004 and, in revised form, August 22, 2005.

2000 Mathematics Subject Classification. Primary 35K10, 35B40.

Key words and phrases. Parabolic operator, rapidly decaying solution, modulus of continuity, Osgood condition. 
Several results (see e.g. [2, 5, 8, 9]) were obtained, relating the decay of $C_{1}(t), C_{2}(t)$ and $\left\|\nabla_{x} a_{i j}(t, \cdot)\right\|_{L^{\infty}}$ to that of the solutions of (1.3). Some years later, Agmon and Nirenberg [1] reconsidered the whole matter by an abstract point of view and proved a general result for inequalities of the form

$$
\left\|\frac{d u}{d t}+A(t) u\right\| \leq \Phi(t)\|u\|
$$

in a Banach space $X$.

Without entering into technical details, we note that there is a common feature in all the above mentioned results: at a certain point one needs to perform some integration by parts, and this requires some (kind of) differentiability of the coefficients with respect to $t$. That a certain amount of regularity was actually necessary in order to get lower bounds for the solutions became clear thanks to a well-known example of Miller [7]. He exhibited a parabolic operator whose coefficients are Hölder continuous of order $1 / 6$ with respect to $t$ and which possesses solutions vanishing within a finite time.

The aim of this paper is the following: for a parabolic inequality of the form (1.3), find the minimal regularity of the coefficients $a_{i j}$ with respect to $t$, ensuring that no solution, except the trivial one, can tend to zero faster than any exponential.

We prove that a sufficient regularity condition is given in terms of a modulus of continuity satisfying the so-called Osgood condition. The counterexample contained in [3] shows that this condition is optimal. The main result (Theorem 1 below) is a consequence of a Carleman estimate in which the weight function depends on the modulus of continuity; such kinds of weight functions in Carleman estimates were introduced by Tarama [10] in the study of second order elliptic operators.

In order to make the presentation simpler, we consider an equation whose coefficients are independent of the space variable $x$. The general case can be recovered by the same microlocal approximation procedure exploited in 3 .

Let $a$ be a continuous function defined on $\mathbb{R}^{+}$such that

$$
\Lambda_{0}^{-1} \leq a(t) \leq \Lambda_{0}
$$

for some $\Lambda_{0} \geq 1$ and for all $t \in \mathbb{R}^{+}$. Let $\varphi$ be a positive function in $L^{1}\left(\mathbb{R}^{+}\right)$. Let $u$ be a function defined on $\mathbb{R}_{t}^{+} \times \mathbb{R}_{x}$ such that

$$
u \in L_{\mathrm{loc}}^{2}\left(\mathbb{R}_{t}^{+}, H^{2}\left(\mathbb{R}_{x}\right)\right) \cap H_{\mathrm{loc}}^{1}\left(\mathbb{R}_{t}^{+}, L^{2}\left(\mathbb{R}_{x}\right)\right)
$$

and

$$
\left\|u_{t}(t, \cdot)-a(t) u_{x x}(t, \cdot)\right\|_{L^{2}\left(\mathbb{R}_{x}\right)}^{2} \leq \varphi(t)\|u(t, \cdot)\|_{H^{1}\left(\mathbb{R}_{x}\right)}^{2}
$$

for a.e. $t \in \mathbb{R}^{+}$. A function $u$ satisfying the conditions (1.6) and (1.7) is called a rapidly decaying solution to (1.7) if for all $\lambda>0$,

$$
\lim _{t \rightarrow+\infty} e^{\lambda t}\|u(t, \cdot)\|_{H^{1}\left(\mathbb{R}_{x}\right)}=0 .
$$

Let $\mu$ be a modulus of continuity, i.e. $\mu$ is a function defined on $\mathbb{R}^{+}$with values in $\mathbb{R}^{+}$such that $\mu$ is continuous, increasing, concave and $\mu(0)=0$. A modulus of continuity $\mu$ is said to satisfy the Osgood condition if

$$
\int_{0}^{1} \frac{1}{\mu(s)} d s=+\infty
$$

Now we can state our main result. 
Theorem 1. Let $\mu$ be a modulus of continuity satisfying the Osgood condition. Suppose that there exists a positive function $\psi$ in $L^{1}\left(\mathbb{R}^{+}\right) \cap L^{\infty}\left(\mathbb{R}^{+}\right)$such that

$$
\sup _{\max \left\{0, t-\frac{1}{2}\right\}<t_{1}<t_{2}<t+\frac{1}{2}} \frac{\left|a\left(t_{2}\right)-a\left(t_{1}\right)\right|}{\mu\left(t_{2}-t_{1}\right)} \leq \psi(t)
$$

for a.e. $t \in \mathbb{R}^{+}$.

If $u$ is a rapidly decaying solution to (1.7), then $u \equiv 0$.

The counterexample alluded to above is given by the following.

Theorem 2. Let $\mu$ be a modulus of continuity which does not satisfy the Osgood condition. Then there exists $l \in C\left(\mathbb{R}_{t}\right)$ with $1 / 2 \leq l(t) \leq 3 / 2$ for all $t \in \mathbb{R}_{t}$ and

$$
\sup _{\substack{0<\left|t_{1}-t_{2}\right|<1 \\ t_{1}, t_{2} \in \mathbb{R}_{t}}} \frac{\left|l\left(t_{2}\right)-l\left(t_{1}\right)\right|}{\mu\left(t_{2}-t_{1}\right)}<\infty
$$

and there exists $u, b_{1}, b_{2}, c \in C_{b}^{\infty}\left(\mathbb{R}_{t} \times \mathbb{R}_{x}^{2}\right)$ with supp $u=\{t \leq 1\}$ such that

$$
\partial_{t} u-\left(\partial_{x_{1}}^{2} u+l \partial_{x_{2}}^{2} u\right)+b_{1} \partial_{x_{1}} u+b_{2} \partial_{x_{2}} u+c u=0 \quad \text { in } \mathbb{R}_{t} \times \mathbb{R}_{x}^{2} .
$$

The proof of Theorem 2 is contained in our previous paper [3].

\section{Proof of Theorem 1}

First of all we remark that it is not restrictive to suppose that $\int_{t_{1}}^{t_{2}} \varphi(s) d s>0$ and $\int_{t_{1}}^{t_{2}} \psi(s) d s>0$ for all $0 \leq t_{1}<t_{2}$. Moreover we will admit without lack of generality that

$$
\int_{0}^{1} \varphi(s) d s \geq 1
$$

Let $\alpha>0$. We set, for $t \geq 0$,

$$
b(t)=\exp \left(-\alpha \int_{0}^{t} \varphi(\eta) d \eta\right) .
$$

Let $\nu$ be a function defined in $[1,+\infty[$ such that

$$
\nu(t)=\int_{1 / t}^{1} \frac{1}{\mu(s)} d s
$$

we remark that (1.9) gives, in particular, $\nu([1,+\infty[)=[0,+\infty[$. For $\gamma>0$ and $\tau \geq 0$ we define

$$
\Psi_{\gamma}(\tau)=\nu^{-1}\left(\gamma \int_{0}^{\tau / \gamma} \psi(s) d s\right)
$$

Finally we set, for $\gamma>0$ and $t \geq 0$,

$$
\Phi_{\gamma}(t)=\int_{0}^{t} \Psi_{\gamma}(\gamma \eta) \frac{1}{b(\eta)}\left(\int_{0}^{\eta} b(s) \varphi(s) d s\right) d \eta
$$


Lemma 1. For all $\alpha>0$ there exists $\gamma_{0}>0$ such that

$$
\begin{aligned}
& \int_{1}^{+\infty} b(t) e^{2 \Phi_{\gamma}(t)}\left\|v_{t}(t, \cdot)-a(t) v_{x x}(t, \cdot)\right\|_{L^{2}\left(\mathbb{R}_{x}\right)}^{2} d t \\
& \geq \frac{\alpha}{\Lambda_{0}} \int_{1}^{+\infty} b(t) \varphi(t) e^{2 \Phi_{\gamma}(t)}\left\|v_{x}(t, \cdot)\right\|_{L^{2}\left(\mathbb{R}_{x}\right)}^{2} d t \\
& \quad+\int_{1}^{+\infty} \Psi_{\gamma}(\gamma t) b(t) \varphi(t) e^{2 \Phi_{\gamma}(t)}\|v(t, \cdot)\|_{L^{2}\left(\mathbb{R}_{x}\right)}^{2} d t
\end{aligned}
$$

for all $\gamma \geq \gamma_{0}$ and for all $v \in L^{2}(] 1,+\infty\left[, H^{2}\left(\mathbb{R}_{x}\right)\right) \cap H^{1}(] 1,+\infty\left[, L^{2}\left(\mathbb{R}_{x}\right)\right)$ with compact support.

Let us show how to prove Theorem 1 from the Carleman estimate (2.6). Let $w$ be a function in $L_{\text {loc }}^{2}(] 1,+\infty\left[, H^{2}\left(\mathbb{R}_{x}\right)\right) \cap H_{\text {loc }}^{1}(] 1,+\infty\left[, L^{2}\left(\mathbb{R}_{x}\right)\right)$ such that $w(t, x)=0$ for all $(t, x) \in] 1,2] \times \mathbb{R}$. Suppose that $w$ satisfies

$$
\lim _{t \rightarrow+\infty} e^{\lambda t}\|w(t, \cdot)\|_{H^{1}\left(\mathbb{R}_{x}\right)}=0
$$

for all $\lambda>0$. We first show that an inequality similar to (2.6) holds for $w$.

Consider $\chi \in C^{\infty}(\mathbb{R})$ with $\chi$ decreasing, $\chi(s)=1$ for $s \leq 1$ and $\chi(s)=0$ for $s \geq 2$, and define $v_{n}(t, x)=\chi(t / n) w(t, x)$. Then

$$
v_{n} \in L^{2}(] 1,+\infty\left[, H^{2}\left(\mathbb{R}_{x}\right)\right) \cap H^{1}(] 1,+\infty\left[, L^{2}\left(\mathbb{R}_{x}\right)\right)
$$

and is compactly supported, so that by (2.6) we deduce

$$
\begin{aligned}
& 2 \int_{1}^{+\infty} b(t) e^{2 \Phi_{\gamma}(t)} \chi^{2}\left(\frac{t}{n}\right)\left\|w_{t}(t, \cdot)-a(t) w_{x x}(t, \cdot)\right\|_{L^{2}}^{2} d t \\
& \geq \frac{\alpha}{\Lambda_{0}} \int_{1}^{+\infty} b(t) \varphi(t) e^{2 \Phi_{\gamma}(t)} \chi^{2}\left(\frac{t}{n}\right)\left\|w_{x}(t, \cdot)\right\|_{L^{2}}^{2} d t \\
& \quad+\int_{1}^{+\infty} \Psi_{\gamma}(\gamma t) b(t) \varphi(t) e^{2 \Phi_{\gamma}(t)} \chi^{2}\left(\frac{t}{n}\right)\|w(t, \cdot)\|_{L^{2}}^{2} d t \\
& \quad-2 \int_{1}^{+\infty} b(t) e^{2 \Phi_{\gamma}(t)}\left(\frac{1}{n} \chi^{\prime}\left(\frac{t}{n}\right)\right)^{2}\|w(t, \cdot)\|_{L^{2}}^{2} d t
\end{aligned}
$$

for all $\gamma \geq \gamma_{0}$. Now remark that $\Psi_{\gamma}(\gamma t) \leq \nu^{-1}\left(\gamma\|\psi\|_{L^{1}\left(\mathbb{R}_{x}\right)}\right)$ for all $\gamma$ and $t$, while $e^{-\alpha\|\varphi\|_{L^{1}\left(\mathbb{R}_{x}\right)} \leq b(t) \leq 1 \text { for all } t \text {. Consequently we have }}$

$$
\Phi_{\gamma}(t) \leq \nu^{-1}\left(\gamma\|\psi\|_{L^{1}}\right)\|\varphi\|_{L^{1}} e^{\alpha\|\psi\|_{L^{1}}} t=C_{\gamma} t
$$

for all $\gamma$ and $t$. Hence, using (2.7) and the fact that $w \in C(] 1,+\infty\left[, H^{1}\left(\mathbb{R}_{x}\right)\right.$ ) (see [6. pp. 18-19]), we deduce that

$$
\begin{gathered}
b(t) \varphi(t) e^{2 \Phi_{\gamma}(t)} \chi^{2}\left(\frac{t}{n}\right)\left\|w_{x}(t, \cdot)\right\|_{L^{2}}^{2} \leq K_{\gamma} \varphi(t), \\
\Psi_{\gamma}(\gamma t) b(t) \varphi(t) e^{2 \Phi_{\gamma}(t)} \chi^{2}\left(\frac{t}{n}\right)\|w(t, \cdot)\|_{L^{2}}^{2} \leq K_{\gamma}^{\prime} \varphi(t)
\end{gathered}
$$

and

$$
b(t) e^{2 \Phi_{\gamma}(t)}\left(\frac{1}{n} \chi^{\prime}\left(\frac{t}{n}\right)\right)^{2}\|w(t, \cdot)\|_{L^{2}}^{2} \leq K_{\gamma}^{\prime \prime} e^{-\tilde{\lambda} t}
$$

for a.e. $t$. Passing to the limit for $n \rightarrow+\infty$, and applying the dominated convergence theorem on the right-hand side and the monotone convergence theorem on 
the left-hand side, we obtain

$$
\begin{aligned}
& \int_{1}^{+\infty} b(t) e^{2 \Phi_{\gamma}(t)}\left\|w_{t}(t, \cdot)-a(t) w_{x x}(t, \cdot)\right\|_{L^{2}}^{2} d t \\
& \geq \frac{\alpha}{2 \Lambda_{0}} \int_{1}^{+\infty} b(t) \varphi(t) e^{2 \Phi_{\gamma}(t)}\left\|w_{x}(t, \cdot)\right\|_{L^{2}}^{2} d t \\
& \quad+\frac{1}{2} \int_{1}^{+\infty} \Psi_{\gamma}(\gamma t) b(t) \varphi(t) e^{2 \Phi_{\gamma}(t)}\|w(t, \cdot)\|_{L^{2}}^{2} d t
\end{aligned}
$$

for all $\gamma \geq \gamma_{0}$.

Now let $u$ be a rapidly decaying solution to (1.7). Let $\theta \in C^{\infty}(\mathbb{R})$ with $\theta$ increasing, $\theta(s)=0$ for $s \leq 2$ and $\theta(s)=1$ for $s \geq 3$. Setting $w(t, x)=\theta(t) u(t, x)$ and applying (2.8) we obtain

$$
\begin{aligned}
& \int_{1}^{3} b(t) e^{2 \Phi_{\gamma}(t)}\left\|(\theta u)_{t}-a(t)(\theta u)_{x x}\right\|_{L^{2}}^{2} d t+\int_{3}^{+\infty} b(t) e^{2 \Phi_{\gamma}(t)}\left\|u_{t}-a(t) u_{x x}\right\|_{L^{2}}^{2} d t \\
& =\int_{1}^{+\infty} b(t) e^{2 \Phi_{\gamma}(t)}\left\|w_{t}-a(t) w_{x x}\right\|_{L^{2}}^{2} d t \\
& \geq \frac{\alpha}{2 \Lambda_{0}} \int_{1}^{+\infty} b(t) \varphi(t) e^{2 \Phi_{\gamma}(t)}\left\|w_{x}\right\|_{L^{2}}^{2} d t+\frac{1}{2} \int_{1}^{+\infty} \Psi_{\gamma}(\gamma t) b(t) \varphi(t) e^{2 \Phi_{\gamma}(t)}\|w\|_{L^{2}}^{2} d t \\
& \geq \frac{\alpha}{2 \Lambda_{0}} \int_{3}^{+\infty} b(t) \varphi(t) e^{2 \Phi_{\gamma}(t)}\left\|u_{x}\right\|_{L^{2}}^{2} d t+\frac{1}{2} \int_{3}^{+\infty} \Psi_{\gamma}(\gamma t) b(t) \varphi(t) e^{2 \Phi_{\gamma}(t)}\|u\|_{L^{2}}^{2} d t .
\end{aligned}
$$

Hence, also using (1.7) we have

$$
\begin{aligned}
& \int_{1}^{3} b(t) e^{2 \Phi_{\gamma}(t)}\left\|(\theta u)_{t}-a(t)(\theta u)_{x x}\right\|_{L^{2}}^{2} d t \\
& \quad \geq \int_{3}^{+\infty} b(t)\left(\frac{\alpha}{2 \Lambda_{0}}-1\right) \varphi(t) e^{2 \Phi_{\gamma}(t)}\left\|u_{x}\right\|_{L^{2}}^{2} d t \\
& \quad+\int_{3}^{+\infty}\left(\frac{1}{2} \Psi_{\gamma}(\gamma t)-1\right) b(t) \varphi(t) e^{2 \Phi_{\gamma}(t)}\|u\|_{L^{2}}^{2} d t
\end{aligned}
$$

We take $\alpha=2 \Lambda_{0}$. We recall that $\Phi_{\gamma}$ is increasing. Hence

$$
\int_{1}^{3} b(t)\left\|(\theta u)_{t}-a(t)(\theta u)_{x x}\right\|_{L^{2}}^{2} d t \geq \int_{3}^{+\infty}\left(\frac{1}{2} \Psi_{\gamma}(\gamma t)-1\right) b(t) \varphi(t)\|u\|_{L^{2}}^{2} d t
$$

for all $\gamma \geq \gamma_{0}$. Since $\Psi_{\gamma}(\gamma t) \geq \Psi_{\gamma}(\gamma)$ for all $t \geq 1$ we obtain

$$
\int_{3}^{+\infty}\left(\frac{1}{2} \Psi_{\gamma}(\gamma t)-1\right) b(t) \varphi(t)\|u\|_{L^{2}}^{2} d t \geq\left(\frac{1}{2} \Psi_{\gamma}(\gamma)-1\right) \int_{3}^{+\infty} b(t) \varphi(t)\|u\|_{L^{2}}^{2} d t
$$

From (1.9) we deduce that $\lim _{\gamma \rightarrow+\infty} \Psi_{\gamma}(\gamma)=+\infty$ and consequently letting $\gamma$ go to $+\infty$ we obtain that $u(x, t)=0$ in $[3,+\infty[\times \mathbb{R}$. We now apply the backward uniqueness result in [3], and we easily deduce that $u \equiv 0$. 
Let us come to the proof of Lemma 1] Setting $z(t, x)=e^{\Phi_{\gamma}(t)} v(t, x)$ we have

$$
\begin{aligned}
& \int_{1}^{+\infty} b(t) e^{2 \Phi_{\gamma}(t)}\left\|v_{t}(t, \cdot)-a(t) v_{x x}(t, \cdot)\right\|_{L^{2}\left(\mathbb{R}_{x}\right)}^{2} d t \\
& =\int_{1}^{+\infty} b(t)\left\|z_{t}(t, \cdot)-a(t) z_{x x}(t, \cdot)-\Phi_{\gamma}^{\prime}(t) z(t, \cdot)\right\|_{L^{2}}^{2} d t \\
& =\int_{1}^{+\infty} \int_{\mathbb{R}_{\xi}} b(t)\left|\hat{z}_{t}(t, \xi)\right|^{2} d \xi d t+\int_{1}^{+\infty} \int_{\mathbb{R}_{\xi}} b(t)\left(a(t) \xi^{2}-\Phi_{\gamma}^{\prime}(t)\right)^{2}|\hat{z}(t, \xi)|^{2} d \xi d t \\
& +2 \Re \int_{1}^{+\infty} \int_{\mathbb{R}_{\xi}} b(t)\left(a(t) \xi^{2}-\Phi_{\gamma}^{\prime}(t)\right) \hat{z}_{t}(t, \xi) \bar{z}(t, \xi) d \xi d t,
\end{aligned}
$$

where $\hat{z}$ denotes the Fourier transform of $z$ with respect to the $x$ variable. We compute the second part of the last term of the above inequality, and we obtain

$$
\begin{aligned}
& -2 \Re \int_{1}^{+\infty} \int_{\mathbb{R}_{\xi}} b(t) \Phi_{\gamma}^{\prime}(t) \hat{z}_{t}(t, \xi) \overline{\hat{z}(t, \xi)} d \xi d t \\
& =\int_{1}^{+\infty} \Psi_{\gamma}(\gamma t) b(t) \varphi(t)\|z(t, \cdot)\|_{L^{2}}^{2} d t \\
& \quad+\int_{1}^{+\infty} \int_{\mathbb{R}_{\xi}} \gamma \Psi_{\gamma}^{\prime}(\gamma t)\left(\int_{0}^{t} b(s) \varphi(s) d s\right)|\hat{z}(t, \xi)|^{2} d \xi d t .
\end{aligned}
$$

It remains to estimate the quantity

$$
2 \Re \int_{1}^{+\infty} \int_{\mathbb{R}_{\xi}} b(t) a(t) \xi^{2} \hat{z}_{t}(t, \xi) \overline{\hat{z}(t, \xi)} d \xi d t .
$$

Since $a$ is not Lipschitz-continuous and consequently we cannot integrate by parts, we exploit the approximation technique developed in [3]. Let $\rho \in C_{0}^{\infty}(\mathbb{R})$ with $\operatorname{supp} \rho \subseteq[-1 / 2,1 / 2], \int_{\mathbb{R}} \rho(s) d s=1$ and $\rho(s) \geq 0$ for all $s \in \mathbb{R}$. We set

$$
a_{\varepsilon}(t)=\int_{\mathbb{R}} a(s) \frac{1}{\varepsilon} \rho\left(\frac{t-s}{\varepsilon}\right) d s,
$$

where $a$ has been extended to $\mathbb{R}$ setting $a(t)=a(0)$ for all $t \leq 0$. We obtain that there exists $C_{0}>0$ such that

$$
\left|a_{\varepsilon}(t)-a(t)\right| \leq \mu(\varepsilon) \psi(t)
$$

and

$$
\left|a_{\varepsilon}^{\prime}(t)\right| \leq C_{0} \frac{\mu(\varepsilon)}{\varepsilon} \psi(t)
$$

for all $\varepsilon \in] 0,1 / 2\left[\right.$ and for a.e. $t \in \mathbb{R}^{+}$. Hence

$$
\begin{aligned}
& 2 \Re \int_{1}^{+\infty} \int_{\mathbb{R}_{\xi}} b(t) a(t) \xi^{2} \hat{z}_{t}(t, \xi) \overline{\hat{z}(t, \xi)} d \xi d t \\
& =2 \Re \int_{1}^{+\infty} \int_{\mathbb{R}_{\xi}} b(t) a_{\varepsilon}(t) \xi^{2} \hat{z}_{t}(t, \xi) \overline{\hat{z}(t, \xi)} d \xi d t \\
& \quad+2 \Re \int_{1}^{+\infty} \int_{\mathbb{R}_{\xi}} b(t)\left(a(t)-a_{\varepsilon}(t)\right) \xi^{2} \hat{z}_{t}(t, \xi) \overline{\hat{z}(t, \xi)} d \xi d t .
\end{aligned}
$$


We have

$$
\begin{aligned}
& 2 \Re \int_{1}^{+\infty} \int_{\mathbb{R}_{\xi}} b(t) a_{\varepsilon}(t) \xi^{2} \hat{z}_{t}(t, \xi) \overline{\hat{z}(t, \xi)} d \xi d t \\
& =-\int_{1}^{+\infty} \int_{\mathbb{R}_{\xi}}\left(b(t) a_{\varepsilon}(t)\right)^{\prime} \xi^{2}|\hat{z}(t, \xi)|^{2} d \xi d t \\
& \quad \geq \int_{1}^{+\infty} \int_{\mathbb{R}_{\xi}} b(t)\left(\alpha \varphi(t) a_{\varepsilon}(t)-\left|a_{\varepsilon}^{\prime}(t)\right|\right) \xi^{2}|\hat{z}(t, \xi)|^{2} d \xi d t \\
& \quad \geq \frac{\alpha}{\Lambda_{0}} \int_{1}^{+\infty} b(t) \varphi(t)\left\|z_{x}(t, \cdot)\right\|_{L^{2}}^{2} d t \\
& \quad-C_{0} \int_{1}^{+\infty} \int_{\mathbb{R}_{\xi}} b(t) \psi(t) \frac{\mu(\varepsilon)}{\varepsilon} \xi^{2}|\hat{z}(t, \xi)|^{2} d \xi d t
\end{aligned}
$$

and

$$
\begin{aligned}
& 2 \Re \int_{1}^{+\infty} \int_{\mathbb{R}_{\xi}} b(t)\left(a(t)-a_{\varepsilon}(t)\right) \xi^{2} \hat{z}_{t}(t, \xi) \overline{\hat{z}(t, \xi)} d \xi d t \\
& \geq-\int_{1}^{+\infty} \int_{\mathbb{R}_{\xi}} b(t)\left|\hat{z}_{t}(t, \xi)\right|^{2} d \xi d t-\int_{1}^{+\infty} \int_{\mathbb{R}_{\xi}} b(t) \psi^{2}(t) \mu^{2}(\varepsilon) \xi^{4}|\hat{z}(t, \xi)|^{2} d \xi d t .
\end{aligned}
$$

Putting all these inequalities together it is easy to see that (2.6) will be a consequence of the following claim: for all $\alpha>0$ there exist $\gamma_{0}>0$ and a function $\mathbb{R} \rightarrow] 0,1 / 2\left[, \xi \mapsto \varepsilon_{\xi}\right.$ such that

$$
\begin{aligned}
\int_{1}^{+\infty} & \int_{\mathbb{R}_{\xi}}\left(b(t)\left(a(t) \xi^{2}-\Phi_{\gamma}^{\prime}(t)\right)^{2}+\gamma \Psi_{\gamma}^{\prime}(\gamma t) \int_{0}^{t} b(s) \varphi(s) d s\right)|\hat{z}(t, \xi)|^{2} d \xi d t \\
& -\int_{1}^{+\infty} \int_{\mathbb{R}_{\xi}} b(t) \psi(t)\left(C_{0} \frac{\mu\left(\varepsilon_{\xi}\right)}{\varepsilon_{\xi}} \xi^{2}+\psi(t) \mu^{2}\left(\varepsilon_{\xi}\right) \xi^{4}\right)|\hat{z}(t, \xi)|^{2} d \xi d t \geq 0
\end{aligned}
$$

for all $\gamma \geq \gamma_{0}$ and for all $z(t, x)=e^{\Phi_{\gamma}(t)} v(t, x)$, provided $v \in L^{2}(] 1,+\infty\left[, H^{2}\left(\mathbb{R}_{x}\right)\right) \cap$ $H^{1}(] 1,+\infty\left[, L^{2}\left(\mathbb{R}_{x}\right)\right)$ is compactly supported.

From (2.3) and (2.4) we have that

$$
\Psi_{\gamma}^{\prime}(\gamma t)=\Psi_{\gamma}^{2}(\gamma t) \mu\left(\frac{1}{\Psi_{\gamma}(\gamma t)}\right) \psi(t)
$$

The concavity of $\mu$ implies that the function $\sigma \mapsto \sigma \mu(1 / \sigma)$ is increasing on $[1,+\infty[$ and consequently the function $\sigma \mapsto \sigma^{2} \mu(1 / \sigma)$ is increasing, and $\sigma^{2} \mu(1 / \sigma) \geq \sigma \mu(1)$ for all $\sigma \in[1,+\infty[$. Hence (2.10) gives

$$
\Psi_{\gamma}^{\prime}(\gamma t) \geq \mu(1) \Psi_{\gamma}(\gamma t) \psi(t) \geq \mu(1) \Psi_{\gamma}(\gamma) \psi(t)
$$

for all $t \in[1,+\infty[$. On the other hand from (2.1) and (2.2) we deduce that

$$
\|\varphi\|_{L^{1}} e^{\alpha\|\varphi\|_{L^{1}}} \geq \frac{1}{b(t)} \int_{0}^{t} b(s) \varphi(s) d s \geq 1
$$

for all $t \in\left[1,+\infty\left[\right.\right.$. Finally since $\mu$ is increasing there exists $\xi_{0} \geq \sqrt{2}$ such that

$$
\mu\left(\frac{1}{\xi^{2}}\right) \leq \frac{1}{4 \Lambda_{0}^{2}\|\psi\|_{\infty}\left(C_{0}+\|\psi\|_{\infty} \mu(1)\right)}
$$


for all $\xi$ with $|\xi| \geq \xi_{0}$. Moreover $\lim _{\gamma \rightarrow+\infty} \Psi_{\gamma}(\gamma)=+\infty$ and then there exists $\gamma_{0}>0$ such that

$$
\mu(1) \gamma \Psi_{\gamma}(\gamma) \int_{0}^{1} \varphi(s) d s \geq\left(C_{0}+\|\psi\|_{\infty} \mu\left(\frac{1}{\xi_{0}^{2}}\right)\right) \mu\left(\frac{1}{\xi_{0}^{2}}\right) \xi_{0}^{4}
$$

for all $\gamma \geq \gamma_{0}$. It is not restrictive to also suppose that

(2.15) $\xi_{0} \geq 2 \Lambda_{0}\|\varphi\|_{L^{1}} e^{\alpha\|\varphi\|_{L^{1}}} \quad$ and $\quad \gamma_{0} \geq 4 \Lambda_{0}^{2}\|\varphi\|_{L^{1}}^{2} e^{2 \alpha\|\varphi\|_{L^{1}}}\left(C_{0}+\|\psi\|_{\infty} \mu(1)\right)$.

We set

$$
\varepsilon_{\xi}=\left\{\begin{array}{ccc}
\frac{1}{\xi_{0}^{2}} & \text { if } & |\xi| \leq \xi_{0}, \\
\frac{1}{\xi^{2}} & \text { if } & |\xi| \geq \xi_{0} .
\end{array}\right.
$$

Suppose first $|\xi| \leq \xi_{0}$. From (2.11), (2.12) and (2.14) we have

$$
\begin{aligned}
& \gamma \Psi_{\gamma}^{\prime}(\gamma t) \int_{0}^{t} b(s) \varphi(s) d s \\
& \geq \gamma \mu(1) \Psi_{\gamma}(\gamma) \psi(t) b(t) \int_{0}^{t} \varphi(s) d s \\
& \geq b(t) \psi(t)\left(C_{0}+\|\psi\|_{\infty} \mu\left(\frac{1}{\xi_{0}^{2}}\right)\right) \mu\left(\frac{1}{\xi_{0}^{2}}\right) \xi_{0}^{4} \\
& \quad \geq b(t) \psi(t)\left(C_{0} \frac{\mu\left(\varepsilon_{\xi}\right)}{\varepsilon_{\xi}} \xi^{2}+\psi(t) \mu^{2}\left(\varepsilon_{\xi}\right) \xi^{4}\right)
\end{aligned}
$$

for all $\gamma \geq \gamma_{0}$ and for all $t \in[1,+\infty[$. Consequently

$$
\begin{aligned}
\int_{1}^{+\infty} & \int_{|\xi| \leq \xi_{0}}\left(b(t)\left(a(t) \xi^{2}-\Phi_{\gamma}^{\prime}(t)\right)^{2}+\gamma \Psi_{\gamma}^{\prime}(\gamma t) \int_{0}^{t} b(s) \varphi(s) d s\right)|\hat{z}(t, \xi)|^{2} d \xi d t \\
& -\int_{1}^{+\infty} \int_{|\xi| \leq \xi_{0}} b(t) \psi(t)\left(C_{0} \frac{\mu\left(\varepsilon_{\xi}\right)}{\varepsilon_{\xi}} \xi^{2}+\psi(t) \mu^{2}\left(\varepsilon_{\xi}\right) \xi^{4}\right)|\hat{z}(t, \xi)|^{2} d \xi d t \geq 0
\end{aligned}
$$

for all $\gamma \geq \gamma_{0}$.

Suppose now $|\xi| \geq \xi_{0}$. If $a(t) \xi^{2} \geq 2 \Phi_{\gamma}^{\prime}(t)$, then

$$
b(t)\left(a(t) \xi^{2}-\Phi_{\gamma}^{\prime}(t)\right)^{2} \geq b(t) \frac{a^{2}(t)}{4} \xi^{4} \geq b(t) \frac{1}{4 \Lambda_{0}^{2}} \xi^{4} .
$$

As a consequence, from (2.13), we have that

$$
\begin{gathered}
b(t) \psi(t)\left(C_{0} \frac{\mu\left(\varepsilon_{\xi}\right)}{\varepsilon_{\xi}} \xi^{2}+\psi(t) \mu^{2}\left(\varepsilon_{\xi}\right) \xi^{4}\right) \\
=b(t) \psi(t)\left(C_{0} \mu\left(\frac{1}{\xi^{2}}\right) \xi^{4}+\psi(t) \mu^{2}\left(\frac{1}{\xi^{2}}\right) \xi^{4}\right) \\
\leq b(t)\|\psi\|_{\infty}\left(C_{0}+\|\psi\|_{\infty} \mu(1)\right) \mu\left(\frac{1}{\xi^{2}}\right) \xi^{4} \\
\leq b(t) \frac{1}{4 \Lambda_{0}^{2}} \xi^{4} \leq b(t)\left(a(t) \xi^{2}-\Phi_{\gamma}^{\prime}(t)\right)^{2} .
\end{gathered}
$$

If $a(t) \xi^{2} \leq 2 \Phi_{\gamma}^{\prime}(t)$, then (1.5), (2.5) and (2.12) imply that

$$
\Psi_{\gamma}(\gamma t) \geq \frac{\xi^{2}}{2 \Lambda_{0}\|\varphi\|_{L^{1}} e^{\alpha\|\varphi\|_{L^{1}}}} .
$$


From (2.10) we infer

$$
\begin{gathered}
\Psi_{\gamma}^{\prime}(\gamma t) \geq \frac{\xi^{4}}{4 \Lambda_{0}^{2}\|\varphi\|_{L^{1}}^{2} e^{2 \alpha\|\varphi\|_{L^{1}}}} \mu\left(\frac{2 \Lambda_{0}\|\varphi\|_{L^{1}} e^{\alpha\|\varphi\|_{L^{1}}}}{\xi^{2}}\right) \psi(t) \\
\geq \frac{\xi^{4}}{4 \Lambda_{0}^{2}\|\varphi\|_{L^{1}}^{2} e^{2 \alpha\|\varphi\|_{L^{1}}}} \mu\left(1 / \xi^{2}\right) \psi(t) .
\end{gathered}
$$

Then

$$
\gamma \Psi_{\gamma}^{\prime}(\gamma t) \int_{0}^{t} b(s) \varphi(s) d s \geq b(t) \psi(t)\left(C_{0} \frac{\mu\left(\varepsilon_{\xi}\right)}{\varepsilon_{\xi}} \xi^{2}+\psi(t) \mu^{2}\left(\varepsilon_{\xi}\right) \xi^{4}\right)
$$

for all $\gamma \geq \gamma_{0}$. Finally, (2.16) and (2.17) give

$$
\begin{aligned}
\int_{1}^{+\infty} & \int_{|\xi| \geq \xi_{0}}\left(b(t)\left(a(t) \xi^{2}-\Phi_{\gamma}^{\prime}(t)\right)^{2}+\gamma \Psi_{\gamma}^{\prime}(\gamma t) \int_{0}^{t} b(s) \varphi(s) d s\right)|\hat{z}(t, \xi)|^{2} d \xi d t \\
& -\int_{1}^{+\infty} \int_{|\xi| \geq \xi_{0}} b(t) \psi(t)\left(C_{0} \frac{\mu\left(\varepsilon_{\xi}\right)}{\varepsilon_{\xi}} \xi^{2}+\psi(t) \mu^{2}\left(\varepsilon_{\xi}\right) \xi^{4}\right)|\hat{z}(t, \xi)|^{2} d \xi d t \geq 0
\end{aligned}
$$

for all $\gamma \geq \gamma_{0}$. The proof of Lemma 1 is complete.

\section{REFERENCES}

[1] S. Agmon and L. Nirenberg, Lower bounds and uniqueness theorems for solutions of differential equations in a Hilbert space, Comm. Pure Appl. Math. 20 (1967), 207-229. MR0204829 $(34: 4665)$

[2] P.J. Cohen and M. Lees, Asymptotic decay of solutions of differential inequalities, Pacific J. Math. 11 (1961), 1235-1249. MR0133601 (24:A3427)

[3] D. Del Santo and M. Prizzi, Backward uniqueness for parabolic operators whose coefficients are non-Lipschitz continuous in time, J. Math. Pures Appl. 84 (2005), no. 4, 471-491. MR2133125

[4] P.D. Lax, A stability theorem for solutions of abstract differential equations, and its application to the study of the local behavior of solutions of elliptic equations, Comm. Pure Appl. Math. 9 (1956), 747-766. MR0086991 (19:281a)

[5] M. Lees, Asymptotic behaviour of solutions of parabolic differential inequalities, Canad. J. Math. 14 (1962), 626-631. MR0157116 (28:354)

[6] J. L. Lions and E. Magenes, Nonhomogeneous Boundary Value Problems and Applications I, Springer-Verlag, Berlin, Heidelberg, New York, 1972. MR0350176 (50:2669)

[7] K. Miller, Nonunique continuation for uniformly parabolic and elliptic equations in selfadjoint divergence form with Hölder continuous coefficients, Arch. Rational Mech. Anal. 54 (1974), 105-117. MR0342822(49:7566)

[8] H. Ogawa, Lower bounds for solutions of parabolic differential inequalities, Canad. J. Math. 19 (1967), 667-672. MR0255967(41:627)

[9] M.H. Protter, Properties of solutions of parabolic equations and inequalities, Canad. J. Math. 13 (1961), 331-345. MR0153982 (27:3943)

[10] S. Tarama, Local uniqueness in the Cauchy problem for second order elliptic equations with non-Lipschitzian coefficients, Publ. Res. Inst. Math. Sci. 33 (1997), no. 1, 167-188. MR 1442496 (98g:35053)

Dipartimento di Matematica e Informatica, Università di Trieste, Via Valerio 12/1, 34127 Trieste, Italy

E-mail address: delsanto@univ.trieste.it

Dipartimento di Matematica e Informatica, Università di Trieste, Via Valerio 12/1, 34127 Trieste, Italy

E-mail address: prizzi@dsm.univ.trieste.it 